\title{
Exponential and power-law expansion of the Universe from the type IIB matrix model
}

\author{
Asato Tsuchiya \\ Department of Physics, Shizuoka University \\ 836 Ohya, Suruga-ku, Shizuoka 422-8529, Japan \\ E-mail: tsuchiya.asato@shizuoka.ac.jp
}

\begin{abstract}
It was shown recently that expanding (3+1)-dimensional Universe emerges dynamically in the Lorentzian version of the type IIB matrix model. Here, we consider two simplified models, which are expected to describe the expanding behaviors at early times and late times, respectively. By performing large-scale computations, we observe that exponential expansions in the former model and power-law expansion in the latter model, which are reminiscent of the expansion in the standard cosmology.
\end{abstract}

Proceedings of the Corfu Summer Institute 2015 "School and Workshops on Elementary Particle Physics and Gravity"

1-27 September 2015

Corfu, Greece 


\section{Introduction}

Superstring theory is a promising candidate for a unified theory including quantum gravity. There are numerous perturbatively (meta-)stable vacua, and perturbation theory does not resolve the cosmic singularity. To elucidate the true nonperturbative vacuum and resolve the cosmic singularity, one needs a nonperturbative formulation. The type IIB matrix model [1] is expected to provide such a formulation.

In [2], Kim, Nishimura and the present author showed by Monte Carlo simulation that expanding (3+1)-dimensional Universe emerges dynamically in the Lorentzian version of the type IIB matrix model. The behavior of expansion is consistent with an exponential expansion [3]. Here only very initial time of the Universe is observed, and simulation at larger matrix size is needed to see later times. However, computational cost is higher for larger matrix size.

Hence, in this talk, we propose and study two simplified models of type IIB matrix model. One is called the VDM model [4], and the other is called the bosonic model [5]. The former is expected to effectively describe the expanding behavior at early times, while the latter that at late times. By performing large scale parallel computations, we reach around twenty times larger matrix size than the case of the original model. In the VDM model, we observe an exponential expansion [6], which is reminiscent of the inflation. On the other hand, in the bosonic model, we observe a power-law expansion [5], which is reminiscent of the expansion in the radiation dominant era of the Universe.

\section{Type IIB matrix model}

The action of the type IIB matrix model [1] is

$$
\begin{aligned}
S & =S_{b}+S_{f}, \\
S_{b} & =-\frac{1}{4 g^{2}} \operatorname{Tr}\left(\left[A_{\mu}, A_{v}\right]\left[A^{\mu}, A^{v}\right]\right), \\
S_{f} & =-\frac{1}{2 g^{2}} \operatorname{Tr}\left(\psi_{\alpha}\left(\mathscr{C} \Gamma^{\mu}\right)_{\alpha \beta}\left[A_{\mu}, \psi_{\beta}\right]\right),
\end{aligned}
$$

where $A_{\mu}(\mu=0,1, \cdots, 9)$ and $\psi_{\alpha}(\alpha=1, \cdots, 16)$ are $N \times N$ hermitian bosonic and fermionic matrices, respectively. $\Gamma^{\mu}$ are ten-dimensional gamma matrices with the Weyl projection, and $\mathscr{C}$ is the charge conjugation matrix with the Weyl projection. The action is formally obtained by dimensionally reducing ten-dimensional $\mathscr{N}=1$ super Yang-Mills theory to zero.dimension. In this model, the space-time does not exist a priori, but emerges dynamically from the degrees of freedom of matrices.

The evidence that this model provides a nonperturbative formulation of superstring theory is as follows. The action can be viewed as a regularization of the Green-Schwarz action of type IIB string of the Schild type, where $w_{\infty}$ algebra corresponding to the area preserving diffeomorphism on the string worldsheet is regularized by the $\operatorname{su}(N)$ algebra. It is remarkable that this regularization preserves $\mathscr{N}=2$ sypersymmetry in ten dimensions and $S O(9,1)$ symmetry of the Green-Schwarz action, which strongly suggests that the model includes gravity because $\mathscr{N}=2$ sypersymmetry in ten dimensions is maximal. Furthermore, the model is a second quantized theory in the sense that it can represent multi-body system of strings. The interaction between the D-branes in type IIB string 
theory is also reproduced correctly at long distance. It can be shown under reasonable assumptions that light-cone string field theory of type IIB string theory is derived from the Schwinger-Dyson equations for the Wilson loops, which are identified with the creation and annihilation operators of strings [7]. This implies that the model reproduces perturbative expansion in type IIB string theory. In this way, the model is shown to have a connection to type IIB string theory, which corresponds to a point in moduli space of 'superstring theory', if one believes in string duality. By using a formulation that allows one to calculate at strong coupling, one should be able to start anywhere in the moduli space. Because the definition of the type IIB matrix model is not based on the perturbation theory, one can expect the model to give a nonperturbative formulation of superstring theory.

\section{Lorentzian model}

The partition function of the Lorentzian version of the type IIB matrix model is defined by

$$
Z=\int d A d \psi e^{i S}=\int d A \operatorname{Pf}(A) e^{i S_{b}}
$$

where the factor ' $i$ ' in the exponent is motivated from the fact that the metric on the string worldsheet should also has the Lorentzian signature, and $\operatorname{Pf}(A)$ is the Pfaffian coming from the integral over the fermionic matrices The bosonic action is decomposed as

$$
S_{b}=-\frac{1}{4 g^{2}} \operatorname{Tr}\left(-2\left[A_{0}, A_{i}\right]^{2}+\left[A_{i}, A_{j}\right]^{2}\right),
$$

which indicates that it is unbounded since $-\operatorname{Tr}\left[A_{0}, A_{i}\right]^{2}$ and $-\operatorname{Tr}\left[A_{i}, A_{j}\right]^{2}$ are positive definite. Due to this unboundedness, we need to introduce the IR cutoffs:

$$
\frac{1}{N} \sum_{i=1}^{9} \operatorname{Tr}\left(A_{i}^{2}\right) \leq L^{2}, \quad \frac{1}{N} \operatorname{Tr}\left(A_{0}^{2}\right) \leq \kappa \frac{1}{N} \sum_{i=1}^{9} \operatorname{Tr}\left(A_{i}^{2}\right),
$$

where we put $L=1$ without loss of generality since the action is homogeneous in $A_{\mu}$ and $\psi$ (for details, see [4]), and we take the $\kappa \rightarrow \infty$ limit as $N \rightarrow \infty$.

We can fix $\mathrm{SU}(N)$ symmetry $A_{\mu} \rightarrow U A_{\mu} U^{\dagger}$ where $U \in \mathrm{SU}(N)$. We take a gauge in which $A_{0}=\operatorname{diag}\left(\alpha_{1}, \alpha_{2}, \cdots, \alpha_{N}\right)$ with $\alpha_{1}<\alpha_{2}<\cdots<\alpha_{N}$. Note that the values of $\alpha_{a}(a=1, \cdots, N)$ are determined dynamically. It turns out that $A_{i}(i=1, \cdots, 9)$ have a band-diagonal structure in this gauge. Namely, there is a certain integer $n$ such that the magnitude of $\left(A_{i}\right)_{a b}$ for $|a-b|>n$ is much smaller than that for $|a-b|<n$. Thus it is natural to define $n \times n$ matrices

$$
\bar{A}_{i}(t)_{I J}=\left(A_{i}\right)_{v+I, v+J}
$$

where $I, J=1, \cdots, n$ and $v=0,1, \cdots, N-n$, and $t$ is the time corresponding to the $n \times n$ block $\left(A_{i}\right)_{v+I, v+J}$ :

$$
t=\frac{1}{n} \sum_{I=1}^{n} \alpha_{v+I}
$$




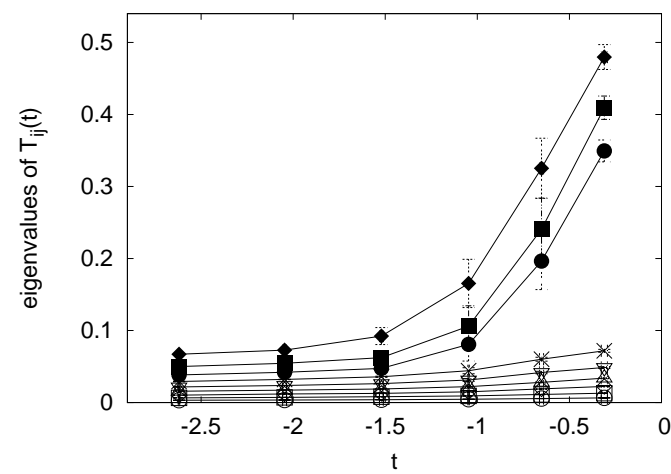

Figure 1: The nine eigenvalues of $T_{i j}(t)$ are plotted against $t$ for $N=16, n=4$ and $\kappa=4.0$. After the critical time $t_{\mathrm{c}}$, three eigenvalues become larger.

The band-diagonal structure should guarantee the locality of time evolution. We interpret $\bar{A}_{i}(t)$ as representing the structure of the space at time $t$. For instance, we define the extent of the space at time $t$ as

$$
R^{2}(t)=\left\langle\frac{1}{n} \sum_{i=1}^{9} \operatorname{tr}\left(\bar{A}_{i}(t)^{2}\right)\right\rangle,
$$

and an analog of the moment of inertia tensor at time $t$ as

$$
T_{i j}(t)=\frac{1}{n} \operatorname{tr}\left(\bar{A}_{i}(t) \bar{A}_{j}(t)\right) .
$$

We denote the nine eigenvalues of $T_{i j}(t)$ by $\lambda_{1}(t), \lambda_{2}(t), \cdots, \lambda_{9}(t)$. Their expectation values represent the extent of space in each of the nine directions at time $t$.

We plot the expectation values of the nine eigenvalues of $T_{i j}(t)$ against $t$ for $N=16, n=4$ and $\kappa=4.0$ in Fig. 10 [2]. We find that three largest eigenvalues of $T_{i j}(t)$ start to grow at the critical time $t_{\mathrm{c}}$, which suggests that the $\mathrm{SO}(9)$ symmetry is spontaneously broken down to $\mathrm{SO}(3)$ after $t_{\mathrm{c}}$.

\section{Simplified models}

The most time-consuming part of the Monte Carlo simulation comes from the Pfaffian, so that we make approximations to it. The fermionic action (2.3) can be decomposed into two terms as

$$
S_{f} \propto \operatorname{Tr}\left(\psi_{\alpha}\left(\mathscr{C} \Gamma^{0}\right)_{\alpha \beta}\left[A_{0}, \psi_{\beta}\right]\right)+\operatorname{Tr}\left(\psi_{\alpha}\left(\mathscr{C} \Gamma^{i}\right)_{\alpha \beta}\left[A_{i}, \psi_{\beta}\right]\right)
$$

At early times, the second term is less important than the first one, because the expansion of the universe has not proceeded so much. Hence we can omit the second term, so that the Pfaffian is simplified to

$$
\operatorname{Pf}(A) \simeq \Delta^{16}(\alpha)
$$



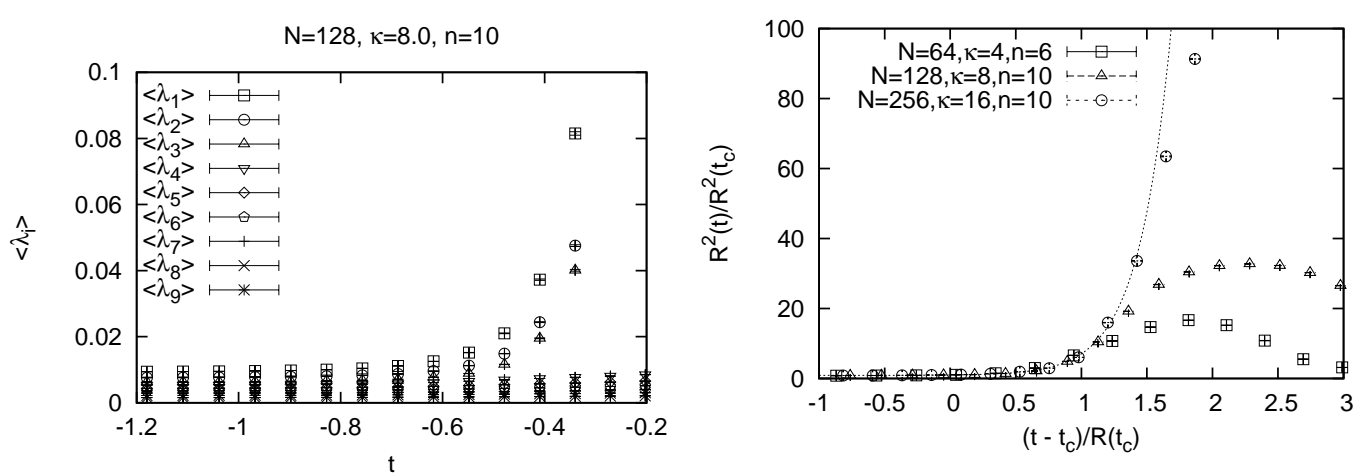

Figure 2: (Left) The nine eigenvalues of $T_{i j}(t)$ are plotted against $t$ for $N=128, \kappa=8.0, n=10$. (Right) The extent of the space $R^{2}(t)$ normalized by $R^{2}\left(t_{\mathrm{c}}\right)$ is plotted against $x=\left(t-t_{\mathrm{c}}\right) / R\left(t_{\mathrm{c}}\right)$ for $N=64,128$ and 256. The dashed line is a fit to $R^{2}(t) / R^{2}\left(t_{\mathrm{c}}\right)=a+(1-a) \exp (b x)$ with $N=256$ for $0 \leq x \leq 1.3$, which gives $a=0.89(3)$ and $b=4.0(3)$.

where $\Delta(\alpha) \equiv \prod_{i>j}\left(\alpha_{i}-\alpha_{j}\right)$ is the van der Monde determinant. Thus we obtain the VDM model, which is expected to effectively describe the expanding behavior at early times.

In Fig. 2(Left), we plot the expectation values of the nine eigenvalues of $T_{i j}(t)$ for $N=128$, $\kappa=8.0, n=10$ [6]. We observe the spontaneous symmetry breaking of $\mathrm{SO}(9)$ to $\mathrm{SO}(3)$ after the critical time $t_{\mathrm{c}}$. In Fig. 2(Right), we plot the extent of the space $R^{2}(t)$ normalized by $R^{2}\left(t_{\mathrm{c}}\right)$ against $x=\left(t-t_{\mathrm{c}}\right) / R\left(t_{\mathrm{c}}\right)$ for $N=64,128$ and 256 [6]. We see that the data for different $N$ scale and that the behavior of $R^{2}(t)$ for $t>t_{\mathrm{c}}$ is fitted to an exponential function. This behavior is reminiscent of the inflation.

On the other hand, at late times, the second term in 4.10 becomes important. As a first approximation, we omit both the first and second terms, namely we ignore all the contribution of the fermions. Thus we obtain the bosonic model, which is expected to effectively describe the expanding behavior at late times. In this model, the IR cutoff for the temporal direction is not needed. We observe that there exist a critical $N_{\mathrm{c}} \sim 110$ such that the symmetry breaking of $\mathrm{SO}(9)$ does not occur for $N<N_{\mathrm{c}}$ while it does for $N>N_{\mathrm{c}}$.

In Fig. 3(Left), we plot the expectation values of the nine eigenvalues of $T_{i j}(t)$ against $t$ for $N=512$ with the block size $n=32$ and observe that the $\mathrm{SO}(9)$ symmetry is spontaneously broken down to $\mathrm{SO}(3)$ after the critical time $t_{\mathrm{c}}$ [5]. In Fig. 3 Right), we plot the extent of space $R^{2}(t)$ normalized by $R^{2}\left(t_{\mathrm{c}}\right)$ against $x=\left(t-t_{\mathrm{c}}\right) / R\left(t_{\mathrm{c}}\right)$ for $N=128,256,384$ and 512 [5]. We observe scaling behavior for different $N$. We see that the behavior of $R^{2}(t)$ at $t>t_{\mathrm{c}}$ can be fitted to an exponential function only for a finite range and that at later times it can be fitted to a linear function, which corresponds to the power-law expansion

$$
R(t) \propto t^{1 / 2}
$$

This behavior is reminiscent of the expansion in the radiation dominant era of the Universe. 

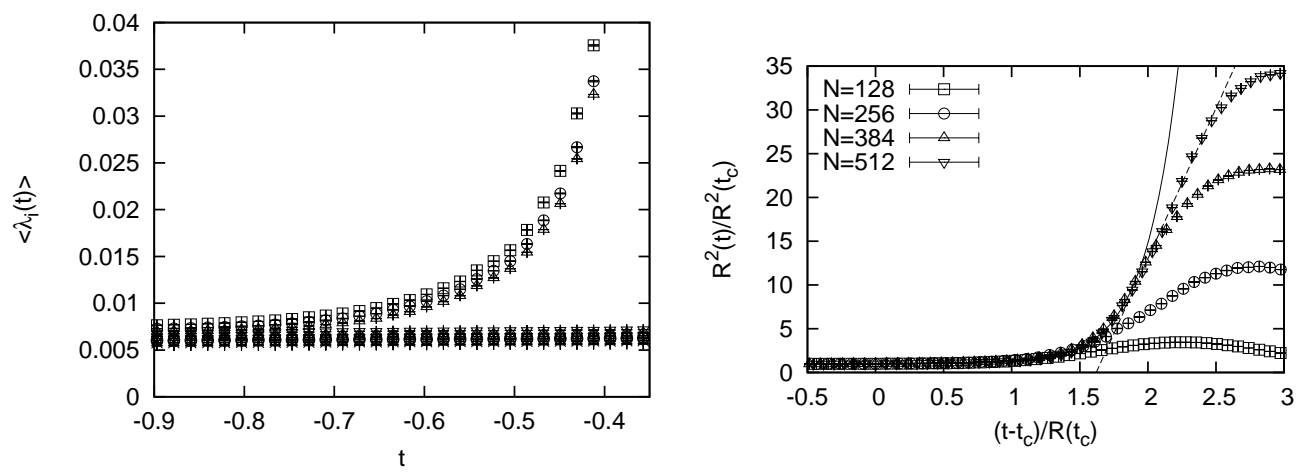

Figure 3: (Left) The expectation values of the nine eigenvalues of $T_{i j}(t)$ are plotted against $t$ for $N=512$, $n=32$. (Right) The extent of space $R^{2}(t)$ normalized by $R^{2}\left(t_{\mathrm{c}}\right)$ is plotted against $x=\left(t-t_{\mathrm{c}}\right) / R\left(t_{\mathrm{c}}\right)$ for $N=128,256,384$ and 512, where the block sizes are $n=20,24,28,32$, respectively. The solid line is a fit of the $N=512$ data to $R^{2}(t) / R^{2}\left(t_{\mathrm{c}}\right)=a+(1-a) \exp (b x)$ for $1.0 \leq x \leq 1.85$, which gives $a=0.9957(5)$ and $b=4.03(7)$. The dashed line is a fit of the $N=512$ data to $R^{2}(t) / R^{2}\left(t_{\mathrm{c}}\right)=c x+d$ for $1.85 \leq x \leq 2.5$, which gives $c=34.3(6)$ and $d=-55(1)$.

\section{Conclusion and discussion}

In this talk, we showed the Monte Carlo results for the type IIB matrix model and the two simplified models, the VDM model and the bosonic model. We are allowed to simulate at large matrix size in the simplified models due to the simplification of the Pfaffian and the large-scale parallel computation.

It turned out that the spontaneous symmetry breaking of $\mathrm{SO}(9)$ to $\mathrm{SO}(3)$ occurs after a critical time in the original and simplified models. Namely only three spatial directions out of nine directions start to grow after the critical time. In the VDM model, we see that the expansion is an exponential one, which is reminiscent of the inflation. In the bosonic model, we observe that the expansion can be fitted at late times to a power-law one, $R(t) \sim t^{\frac{1}{2}}$, which is reminiscent of the expansion in the radiation dominant era of the Universe.

We need to see the cutoff independence of the results. In the VDM model, we generalized the IR cutoff (5.1) to

$$
\frac{1}{N} \sum_{i=1}^{9} \operatorname{Tr}\left(A_{i}^{2 p}\right) \leq L^{2 p}, \quad \frac{1}{N} \operatorname{Tr}\left(A_{0}^{2 p}\right) \leq \kappa^{p} \frac{1}{N} \sum_{i=1}^{9} \operatorname{Tr}\left(A_{i}^{2 p}\right),
$$

and obtained universal results for $R(t)^{2}$ in the cases of different $\mathrm{p}$ with $p>1$ [6]. The results are qualitatively the same as the case of $p=1$, but quantitatively different. This implies that the effect of the IR cutoffs disappears for $p>1$ in the large $N$ limit. We expect the same thing to be true for the original model.

It is important to study classical solutions since they are expected to dominate at late times. In [8], we indeed examined classical solutions systematically and found some solutions that are 
cosmologically interesting. (For related recent work, see $[9,10]$.) In [11-14], how the Standard model is realized in the type IIB matrix model was studied based on the idea of intersecting Dbranes. It is also of course important to investigate whether the power-law expansion is seen in the original model. We hope to report progress in these subjects as well as the analysis of the IR cutoff independence in the original model in the near future.

\section{Acknowledgments}

This research used computational resources of the K computer of the HPCI system provided by the AICS through the HPCI System Research Project (Project ID : hp130063). The supercomputer FX10 at University of Tokyo has been used in developing the code for parallel computing. The author was supported in part by Grant-in-Aid for Scientific Research (No. 24540264, 23244057, 15K05046) from Japan Society for the Promotion of Science.

\section{References}

[1] N. Ishibashi, H. Kawai, Y. Kitazawa and A. Tsuchiya, Nucl. Phys. B 498, 467 (1997) doi:10.1016/S0550-3213(97)00290-3 [hep-th/9612115].

[2] S. W. Kim, J. Nishimura and A. Tsuchiya, Phys. Rev. Lett. 108, 011601 (2012) doi:10.1103/PhysRevLett.108.011601 [arXiv:1108.1540 [hep-th]].

[3] Y. Ito, S. W. Kim, J. Nishimura and A. Tsuchiya, PoS LATTICE 2013, 341 (2014) [arXiv:1311.5579 [hep-lat]].

[4] Y. Ito, S. W. Kim, Y. Koizuka, J. Nishimura and A. Tsuchiya, PTEP 2014, no. 8, $083 B 01$ (2014) doi:10.1093/ptep/ptu101 [arXiv:1312.5415 [hep-th]].

[5] Y. Ito, J. Nishimura and A. Tsuchiya, JHEP 1511, 070 (2015) doi:10.1007/JHEP11(2015)070 [arXiv:1506.04795 [hep-th]].

[6] Y. Ito, J. Nishimura and A. Tsuchiya, arXiv:1512.01923 [hep-lat].

[7] M. Fukuma, H. Kawai, Y. Kitazawa and A. Tsuchiya, Nucl. Phys. B 510, 158 (1998) doi:10.1016/S0550-3213(98)81008-0, 10.1016/S0550-3213(97)00584-1 [hep-th/9705128].

[8] S. W. Kim, J. Nishimura and A. Tsuchiya, JHEP 1210, 147 (2012) doi:10.1007/JHEP10(2012)147 [arXiv:1208.0711 [hep-th]].

[9] D. Jurman and H. Steinacker, JHEP 1401, 100 (2014) doi:10.1007/JHEP01(2014)100 [arXiv:1309.1598 [hep-th]].

[10] A. Chaney, L. Lu and A. Stern, Phys. Rev. D 93, no. 6, 064074 (2016) doi:10.1103/PhysRevD.93.064074 [arXiv:1511.06816 [hep-th]].

[11] A. Chatzistavrakidis, H. Steinacker and G. Zoupanos, JHEP 1109, 115 (2011) doi:10.1007/JHEP09(2011)115 [arXiv:1107.0265 [hep-th]].

[12] J. Nishimura and A. Tsuchiya, JHEP 1312, 002 (2013) doi:10.1007/JHEP12(2013)002 [arXiv:1305.5547 [hep-th]].

[13] H. Aoki, J. Nishimura and A. Tsuchiya, JHEP 1405, 131 (2014) doi:10.1007/JHEP05(2014)131 [arXiv:1401.7848 [hep-th]].

[14] H. C. Steinacker and J. Zahn, PTEP 2014, no. 8, 083 B03 (2014) doi:10.1093/ptep/ptu111 [arXiv:1401.2020 [hep-th]]. 